UNIVERSIDADE DE SÃO PAULO

FACULDADE DE MEDICINA DE RIBEIRÃO PRETO

DEPARTAMENTO DE GINECOLOGIA E OBSTETRÍCIA

\title{
ESTUDO HEMODINÂMICO MATERNO FETAL PRÉ E PÓS TRATAMENTO DE CRISE HIPERTENSIVA
}

MARIA RITA DE FIGUEIREDO BAGIO

Ribeirão Preto-SP

2010 
UNIVERSIDADE DE SÃO PAULO

FACULDADE DE MEDICINA DE RIBEIRÃO PRETO

DEPARTAMENTO DE GINECOLOGIA E OBSTETRÍCIA

\section{ESTUDO HEMODINÂMICO MATERNO FETAL PRÉ E PÓS TRATAMENTO DE CRISE HIPERTENSIVA}

MARIA RITA DE FIGUEIREDO BAGIO

Ribeirão Preto-SP

2010 
MARIA RITA DE FIGUEIREDO BAGIO

\section{ESTUDO HEMODINÂMICO MATERNO FETAL PRÉ E PÓS TRATAMENTO DE CRISE HIPERTENSIVA}

Dissertação apresentada à Faculdade de Medicina de Ribeirão Preto da Universidade de São Paulo como requisito para a obtenção do título de Mestre em Ciências Médicas.

Área de concentração: Ginecologia e Obstetrícia

Orientador: Prof. Dr. Ricardo de Carvalho Cavalli

Ribeirão Preto-SP

2010 
Autorizo a reprodução e divulgação total ou parcial deste trabalho, por qualquer meio convencional ou eletrônico, para fins de estudo e pesquisa, desde que citada a fonte.

FICHA CATALOGRÁFICA

Bagio, Maria Rita de Figueiredo

ESTUDO HEMODINÂMICO MATERNO FETAL PRÉ E PÓS TRATAMENTO DE CRISE HIPERTENSIVA

p.XXX : il.; $30 \mathrm{~cm}$

Dissertação de mestrado, apresentada à Faculdade de Medicina de Ribeirão Preto/USP - Área de concentração: Ginecologia e Obstetrícia.

Orientador: Cavalli, Ricardo de Carvalho

1. Hipertensão. 2. Gestação. 3. Doppler. 4. Labetalol. 5. Hidralazina 
Ser feliz é reconhecer que vale a pena viver apesar de todos os desafios, incompreensões e períodos de crise.

Ser feliz é deixar de ser vítima dos problemas e se tornar um autor da própria história. É atravessar desertos fora de si, mas ser capaz de encontrar um oásis no recôndito da sua alma.

É agradecer a Deus a cada manhã pelo milagre da vida.

Ser feliz é não ter medo dos próprios sentimentos.

É saber falar de si mesmo.

É ter coragem para ouvir um "não".

É ter segurança para receber uma crítica, mesmo que injusta.

Pedras no caminho?

Guardo todas, um dia vou construir um castelo...

Fernando Pessoa 


\title{
DEDICO ESSE TRABALHO:
}

\begin{abstract}
À DEUS, que se faz presente na forma de amor em minha vida, guia meus passos e ilumina meus caminhos...
\end{abstract}

Aos meus pais, Ana e José, verdadeiros exemplos de caráter, dignidade e amor incondicional...

Ao meu orientador, Prof. Dr. Ricardo de Carvalho Cavalli, grande amigo e incentivador, que acreditou na minha capacidade e me permitiu desenvolver esse trabalho. 


\section{Agradecimentos}

- Às pacientes e seus filhos que voluntariamente permitiram o desenvolvimento desse trabalho.

- À minha amiga-irmã Claudia Baraldi que esteve ao meu lado em todas as fases desse projeto, sempre disponível, me incentivando nos momentos mais difíceis.

- Ao Dr. Rafael Yano, meu amigo e professor, fonte inesgotável de ensino, referência de obstetrícia para toda a minha vida.

- Aos meus irmãos, Claudia e André, sobrinhos Zé e Lucas e cunhados, Bela e Taba, por junto com meus pais, formarem uma família maravilhosa, onde sempre encontrei amor, apoio e conforto.

- Ao Marcos e a Aninha, por transformarem um relacionamento profissional em uma amizade verdadeira e gratificante que eu pretendo manter por toda a minha vida.

- Às amigas Stefania, Daniela, Claudia e Milena por estarem sempre presentes e tornarem minha vida melhor

- À Elaine por todo o carinho e amizade desde meu primeiro dia no HC.

- Ao Prof. Dr. Nogueira por me transmitir calma e segurança diante das dificuldades encontradas ao longo deste trabalho

- Aos funcionários Reinaldo, Ilza, Ricardo, Suelen e Rosane por me auxiliarem inúmeras vezes em questões administrativas e burocráticas

- Ao Departamento de Ginecologia e Obstetrícia da Faculdade de Medicina de Ribeirão Preto por me proporcionar a oportunidade de desenvolver este trabalho 


\section{SUMÁRIO}

Lista de abreviaturas

Lista de tabelas

Lista de Figuras

Resumo

Abstract

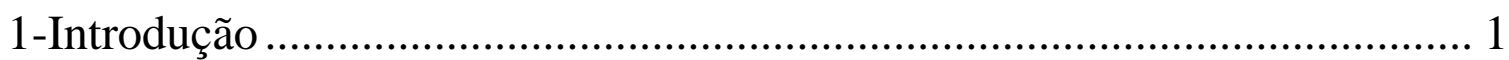

1.1-O estudo Doppler fetal ................................................................ 1

1.2-Síndrome hipertensiva gestacional ............................................. 1

1.3-Avaliação de vitalidade fetal na síndrome hipertensiva gestacional...1

1.4-Doppler e crise hipertensiva ....................................................... 1

1.5-Labetalol como droga anti-hipertensiva ......................................... 1

1.6-Hidralazina como droga anti-hipertensiva ...................................... 2

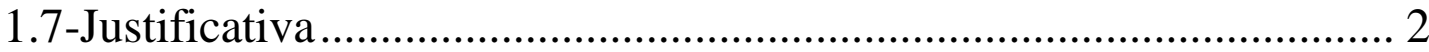

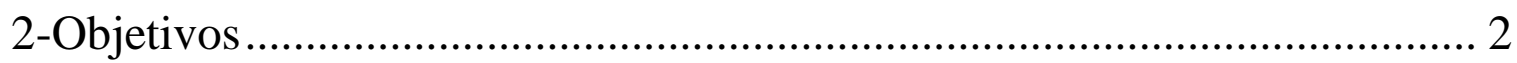

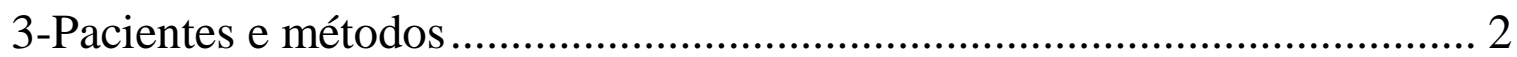

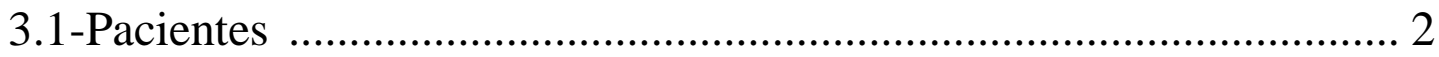




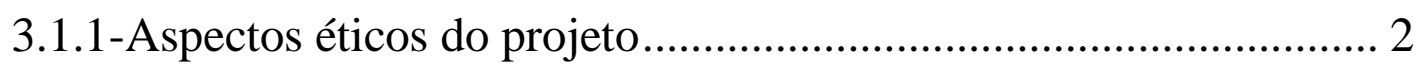

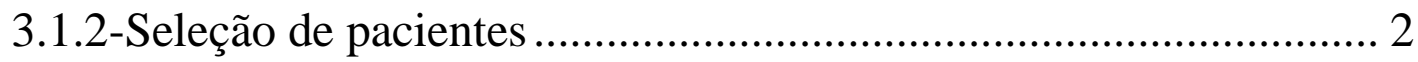

3.1.2.1-Critérios de inclusão ............................................................... 2

3.1.2.2-Critérios de exclusão .............................................................. 2

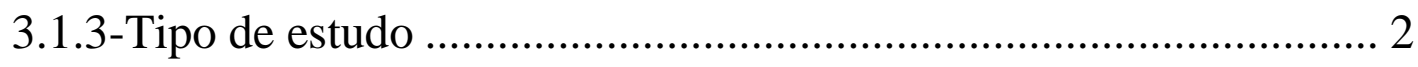

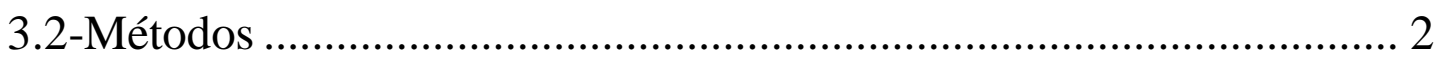

3.2.1-Identificação das pacientes ...................................................... 2

3.2.2-Avaliação perinatal .................................................................... 2

3.2.3-Classificação da hipertensão ........................................................ 2

3.2.4-Avaliação hemodinâmica materna ................................................ 2

3.2.5-Tratamento da crise hipertensiva.............................................. 2

3.2.6-Avaliação ultrassonográfica e dopplervelocimétrica .................... 2

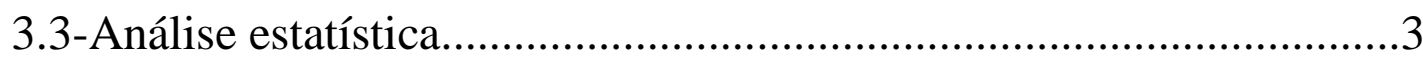

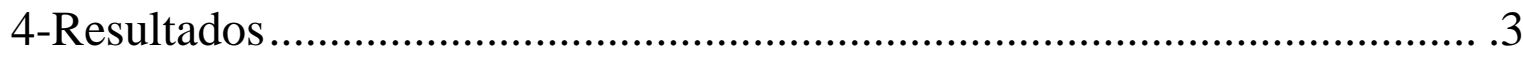

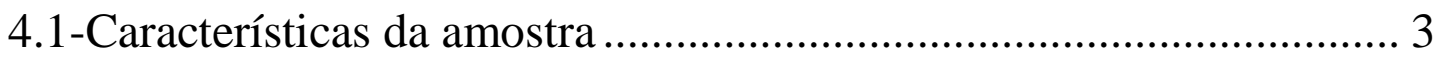

4.2-Avaliação do quadro hipertensivo ...................................................... 3

4.3-Avaliação dopplervelocimétrica ..................................................... 3

4.4-Avaliação de resultados perinatais.......................................................

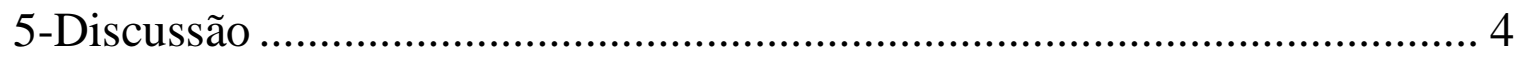




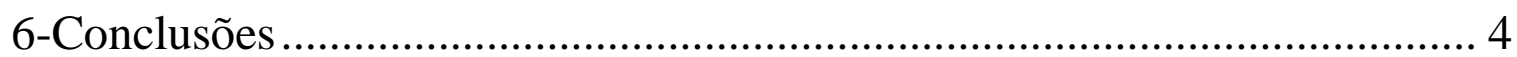

7-Referências bibliográficas ......................................................................... 4

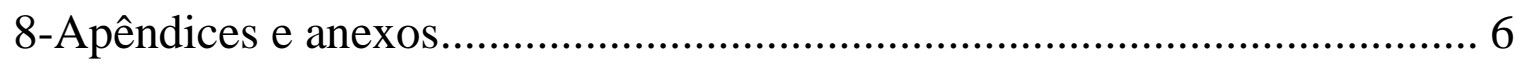




\section{Lista de abreviaturas:}

ACM........................artéria cerebral média

AUm.......................artéria umbilical

AUD........................artéria uterina direita

AUE.........................artéria uterina esquerda

AUs..........................artérias uterinas

Bpm........................batimentos por minuto

FMRP ......................Faculdade de Medicina de Ribeirão Preto

HC.............................Hospital das Clínicas

IP.............................índice de pulsatilidade

IR............................índice de resistência

mg..........................miligramas

mmHg......................milimetros de mercurio

$\mathrm{pCO}_{2}$.....................pressão de dióxido de carbono

$\mathrm{pO}_{2} \ldots \ldots \ldots \ldots \ldots \ldots \ldots \ldots . . .$. pressão de oxigênio

RCIU........................restrição de crescimento intra-útero

SHG.......................sindrome hipertensiva gestacional

USP.............................Universidade de São Paulo 
Lista de Tabelas

Tabela 1. Classificação da hipertensão de acordo com a paridade............................

Tabela 2. Classificação da hipertensão e tratamento da crise hipertensiva..............

Tabela 3. Avaliação laboratorial de gestantes com crise hipertensiva .....................

Tabela 4. Doppler das artérias uterinas: direita versus. esquerda.............................

Tabela 5.Comparação dos parâmetros avaliados inicial versus final........................

Tabela 6.Resultados gestacionais e perinatais 
Lista de Figuras

Figura 1- Estrutura química do labetalol...............................................1

Figura 2- Estrutura química da hidralazina...........................................2

Figura 3. Dopplervelocimetria color e pulsada da artéria uterina............3

Figura 4. Dopplervelocimetria color e pulsada da artéria umbilical........3

Figura 5. Dopplervelocimetria color e pulsada da cerebral média............3 


\section{Resumo:}

Bagio, MRF. Estudo hemodinâmico materno fetal pré e pós-tratamento de crise hipertensiva. 2010. Dissertação (Mestrado) - Faculdade de Medicina de Ribeirão Preto, Universidade de São Paulo, Ribeirão Preto.

\section{Introdução}

A síndrome hipertensiva gestacional (SHG) afeta três milhões de mulheres por ano no mundo e pode resultar em inúmeras perdas fetais e perinatais, além de ser responsável pela morte de uma mulher a cada 6 minutos em todo o mundo.

Durante uma crise hipertensiva na gestação ocorre vasoconstrição arteriolar generalizada e diminuição do fluxo útero placentário. A diminuição de fluxo na artéria uterina ocasiona déficit do aporte de oxigênio nas áreas de troca materno fetal submetendo o feto à regime de hipóxia transitória comprováveis na avaliação hemodinâmica. O labetalol e a hidralazina são anti hipertensivos de primeira escolha na gravidez, uma vez que aumentam o fluxo útero-placentário em decorrência da redução da resistência vascular uterina.

\section{Objetivos}

Avaliar as condições hemodinâmicas materno/fetais durante o período da crise hipertensiva e após seu tratamento com labetalol e/ou hidralazina.

\section{Pacientes e métodos}

Foram avaliadas 18 pacientes com quadro de crise hipertensiva com pressão arterial maior que 160x110mmHg, sem sintomas de eminência de eclâmpsia. Realizou-se estudo dopplervelocimétrico das artérias uterinas direita e esquerda materna, umbilical e cerebral média fetal. 
A avaliação hemodinâmica materno/fetal foi realizada no momento da crise hipertensiva e após o tratamento com labetalol e/ou hidralazina. A análise pós tratamento foi realizada após estabilização da pressão arterial $<150 / 100 \mathrm{mmHg}$.

\section{Resultados}

A média de idade das pacientes foi de 29,11 anos e a idade gestacional média do estudo de 34,5 semanas.

O valor médio da pressão arterial sistólica e diastólica durante a crise hipertensiva foi de 174,71 e $112,35 \mathrm{mmHg}$, respectivamente.

Os parâmetros dopplervelocimétricos avaliados na crise hipertensiva foram: IP da artéria uterina direita 1,24+-0,42; IP da artéria uterina esquerda 1,29+-0,40; IR da artéria cerebral média de 0,78+-0,06 e IR da artéria umbilical de 0,65+-0,12. Após o tratamento da crise hipertensiva a pressão arterial sistólica média foi de $146,47 \mathrm{mmHg}$ e a pressão arterial diastólica média de 87,06mmHg. Após o tratamento da crise hipertensiva, os parâmetros avaliados foram: IP da artéria uterina direita 1,26+-0,37; IP da artéria uterina esquerda 1,37+-0,36; IR da artéria cerebral média de 0,78+-0,09 e IR da artéria umbilical de 0,67+-0,09. Não houve diferenças estatísticas significativas nos parâmetros Doppler quando comprados os valores na crise hipertensiva e após o controle da pressão arterial.

\section{Conclusão}

As gestações de pacientes com síndromes hipertensivas são consideradas de alto risco e, devem ser alvo de observação rigorosa durante o pré-natal. 
A análise dopplervelocimétrica fetal (artéria cerebral média e umbilical) e materna (artérias uterinas) deve ser realizada para avaliação da vitalidade fetal nestas pacientes, inclusive durante de crise hipertensiva.

Este estudo demonstrou não haver diferença na avaliação dos parâmetros dopplervelocimétricos materno e fetal, durante ou após a crise hipertensiva tratada com hidralazina/labetalol.

\section{Palavras-chaves}

Doppler - Hipertensão - Gestação - Labetalol - Hidralazina 


\begin{abstract}
Bagio, MRF. Maternal fetal hemodynamics study before and after treatment of hypertensive crisis. 2010. Dissertação (Mestrado) - Faculdade de Medicina de Ribeirão Preto, Universidade de São Paulo

Introduction
\end{abstract}

The gestational hypertension syndrome affects three million women a year worldwide and can result in a number of fetal losses and perinatal deaths, and was responsible for the death of one woman every 6 minutes throughout the world.

During a hypertensive crisis in pregnancy is widespread arteriolar vasoconstriction and decreased placental flow uterus. The decrease in flow in the uterine artery causes deficit of oxygen supply in the areas of maternal fetal exchange subjecting the fetus to the regime of transient hypoxia in comparable hemodynamic evaluation. Labetalol and hydralazine are antihypertensive drugs of choice in pregnancy, they increase the uteroplacental flow due to the reduction of uterine vascular resistance.

\title{
Objectives
}

To evaluate the conditions maternal-fetal hemodynamics during the hypertensive crisis and after treatment with labetalol and/or hydralazine.

\section{Patients and methods}

Eighteen pregnant women with acute hypertensive were evaluated (blood pressure (BP) > 160x110mmHg, without imminent eclampsia symptoms). This study was performed by Doppler velocimetry of right and left uterine arteries, umbilical artery and fetal middle cerebral artery. 
Maternal-fetal hemodynamic assessment was performed at the time of hypertensive crisis and after treatment with labetalol and/ or hydralazine. Post treatment analysis was performed after blood pressure stabilization $(\mathrm{BP}<150 \times 100 \mathrm{mmHg})$.

\section{Results}

Mean patients age was 29,11 years old and mean gestational age was 34.5 weeks. Mean systolic and diastolic blood pressure during hypertensive crisis was 174.71 and 112.35 $\mathrm{mmHg}$, respectively.

Doppler parameters evaluated in hypertensive crisis were: right uterine artery PI $1.24 \pm$ 0.42; left uterine artery PI $1.29 \pm 0.40$; middle cerebral artery RI $0.78 \pm 0,06$ and umbilical artery RI $0.65 \pm 0.12$. After the hypertensive crisis treatment mean systolic blood pressure was 146.47 $\mathrm{mmHg}$ and diastolic $87.06 \mathrm{mmHg}$. Doppler parameters evaluated after hypertensive crisis were: right uterine artery PI $1.26 \pm 0.37$; left uterine artery PI $1.37 \pm 0.36$; middle cerebral artery RI $0.78 \pm 0,09$ and umbilical artery RI $0.67 \pm 0.09$. No statistical difference was observed in Doppler parameters during hypertensive crisis or after blood pressure control.

\section{Conclusion}

Hypertensive pregnancy disorders are considered high risk to disfavorable perinatal outcome and should be subject to close observation during prenatal by fetal Doppler velocimetry analysis (middle cerebral artery and umbilical cord) and maternal Doppler velocimetry analysis (uterine artery) to assess fetal well being.

This study demonstrated no difference in maternal fetal Doppler parameters during or after hypertensive crisis treated with hydralazine/labetalol.

Keywords

Doppler - Hypertension - Pregnancy - Labetalol - Hydralazine 


\section{1-Introdução}

\section{1-O estudo do Doppler fetal}

A primeira descrição do efeito Doppler foi feita em 1842 por Cristhian Doppler. Na medicina esse método foi introduzido por Satomura, em 1956, ao aplicar o efeito Doppler na obtenção dos movimentos cardíacos, do globo ocular e do sangue nos vasos.

Em 1977, McCallum utilizou o Doppler de onda continua para obter velocidade de fluxo na artéria umbilical após o parto. No mesmo ano, Fitzgerald e Drumm empregaram o Doppler pulsátil para avaliar a velocidade de fluxo sangüíneo nos vasos umbilicais intra-útero, mostrando, pela primeira vez, a característica espectral desses vasos e propiciando o estudo in vivo das circulações útero placentária e feto placentária.

Desde o final dos anos 80, o Doppler colorido é utilizado em várias áreas da medicina, especialmente em obstetrícia. Em 1987, Wladimiroff et al., relataram aumento do índice de pulsatilidade (IP) da artéria umbilical em fetos com restrição de crescimento intra-uterino (RCIU), cuja sensibilidade foi de $89 \%$.

No início dos anos 90, a dopplervelocimetria passou a ser amplamente utilizada na identificação das anormalidades da placenta e do cordão umbilical e nos quadros hipertensivos da gestação (GOLDBERG, 2000).

O método Doopler, através da identificação das modificações hemodinâmicas, tem grande aplicabilidade na avaliação de gestações com comprometimento do fluxo sangüíneo útero placentário, como nos casos de hipertensão induzida pela gravidez, nos gêmeos discordantes e na restrição de crescimento intra-útero (YOSHIMURA et al., 1998). 
A dopplervelocimetria é frequentemente empregada na avaliação materno-fetal em gestações de alto risco, ressaltando as gestações complicadas com hipertensão, contribuindo com a redução da mortalidade perinatal (WESTERGAARD et al., 2001).

Nos estudos da circulação materno-fetal pela dopplervelocimetria, diversos compartimentos são avaliados, dentre eles a artéria uterina, a artéria cerebral média, a artéria umbilical e o ducto venoso, permitindo diagnóstico de alterações da vitalidade fetal desde estágios precoces até comprometimentos extremos (RYCHIK, 2004).

Associado à história materna e às medidas da pressão arterial, o Doppler é um importante preditor de pré-eclâmpsia, atuando como método de screening para pacientes selecionadas (ONWUDIWE et al., 2008).

O método Doppler tornou-se uma ferramenta indispensável na avaliação de gestações de risco para doenças como a pré-eclâmpsia, restrição de crescimento intra-uterino, anemia fetal e anormalidades do cordão umbilical (HOFMAN, 2009).

\section{2- Síndrome hipertensiva gestacional}

As desordens hipertensivas da gravidez afetam aproximadamente $5 \%$ a $8 \%$ das gestantes, contribuindo de forma significativa para o desenvolvimento de complicações materno/fetais graves. A pré-eclâmpsia constitui a causa isolada mais comum de restrição de crescimento intraútero e permanece como um dos principais fatores associados ao aumento da morbidade materna e perinatal (NHBPEP, 2000). 
Em cerca de $70 \%$ dos casos, as alterações da pressão arterial são decorrentes do estado gestacional (hipertensão gestacional, pré-eclâmpsia e eclâmpsia), enquanto os $30 \%$ restantes correspondem à hipertensão pré-existente (AFIFI, 2003).

O Colégio Americano de Obstetras e Ginecologistas (ACOG, 2002) em acordo com o NHBPEP (2000), classifica os transtornos hipertensivos da gestação em: hipertensão arterial crônica, pré-eclâmpsia, pré-eclâmpsia sobreposta à hipertensão arterial crônica e hipertensão gestacional.

É importante distinguir a pré-eclâmpsia, manifestação hipertensiva que ocorre pela primeira vez na gestação, da hipertensão crônica pré-existente. Estas duas desordens são caracterizadas por aumento da pressão arterial, porém com fisiopatologias diferentes e com complicações materno fetais distintas (NHBPEP, 2000).

Dentre os fatores de risco para a pré-eclâmpsia destacam-se os sócio-demográficos (extremos da idade reprodutiva, nível sócio-econômico, etnia), os genéticos, os diretamente ligados à gravidez (gestação múltipla, primeira gestação, antecedente de pré-eclâmpsia) e os antecedentes patológicos pregressos, como obesidade, doença renal crônica, hipertensão arterial crônica, diabetes mellitus e trombofilia (SMITH, 2005).

Além de alterações hemodinâmicas maternas, as desordens hipertensivas da gestação associam-se à insuficiência da circulação útero-placentária e conseqüente restrição do crescimento intra-uterino (DELMIS, 2006).

$\mathrm{Na}$ gestação, a hipertensão arterial pode apresentar desfechos maternos trágicos, tais como insuficiência de múltiplos órgãos, descolamento da placenta e morte, além de agravos fetais como prematuridade, restrição de crescimento e morte perinatal (CAETANO et al., 2004; VASCONCELLOS, 2004). 


\section{3-A valiação da vitalidade fetal na síndrome hipertensiva gestacional}

As chamadas "gestações de alto risco" associam-se a elevadas taxas de morbidade e mortalidade tanto maternas quanto perinatais. A necessidade de avaliação da vitalidade fetal nestas gestações resultou no desenvolvimento de vários métodos propedêuticos nas últimas décadas, contribuindo para redução dos índices de morbidade e mortalidade perinatais (GOLDABER et al., 1991)

Fatores etiológicos de origem materna, fetal e placentária podem prejudicar as trocas gasosas e nutricionais realizadas pela circulação útero placentária, com repercussões sobre o feto que poderá apresentar RCIU associada a elevação das cifras de óbito perinatal. Quanto mais precoce for a agressão sofrida pelo organismo fetal, mais graves serão suas consequiências, contribuindo para aumento dos índices de morbidade e mortalidade perinatais, sofrimento para os familiares e elevados custos para o sistema de saúde. Em algumas situações, a interrupção da gravidez é a conduta preconizada para preservar o feto, retirando-o do ambiente intra-uterino inóspito (NOMURA, 2001).

A restrição de crescimento fetal e a hipóxia intra-útero devem ser diagnosticadas o mais precoce possível, permitindo avaliação seqüencial e resultados perinatais satisfatórios. Neste âmbito, a avaliação dopplervelocimétrica de fetos de gestantes hipertensas é de fundamental importância para diagnóstico e seguimento destas entidades clínicas (BASCHAT et al., 2003). 


\section{4- Doppler e crise hipertensiva}

$\mathrm{O}$ mecanismo de defesa do organismo fetal frente à redução do aporte de nutrientes e $\mathrm{O}_{2}$, por insuficiência placentária, implica em alterações metabólicas e hemodinâmicas capazes de proteger o feto desta agressão. A elevação das concentrações da $\mathrm{pCO}_{2}$ e a redução da $\mathrm{pO}_{2}$ no sangue fetal estimulam os barorreceptores, que desencadeiam a adaptação do organismo fetal determinando vasodilatação das artérias cerebrais, das coronárias e das glândulas supra-renais, fenômeno este denominado centralização fetal.

A vasoconstrição periférica fetal complementa o sentido da preservação dos órgãos fetais considerados mais importantes e relacionados diretamente à sua vitalidade (PEETERS et al., 1979).

A hipóxia tecidual dos órgãos periféricos fetais, conseqüente a vasoconstrição, promove incremento do metabolismo anaeróbico, além de mobilizar glicose por meio da glicogenólise hepática (RURAK \& GRUBER, 1983). Este metabolismo promove acúmulo de ácido láctico que, por não ser volátil e não atravessar a barreira placentária, resulta em desequilíbrio ácidobásico com redução do pH do sangue fetal à medida que os sistemas tampões são consumidos. Esta resposta metabólica está diretamente relacionada à duração do evento hipoxêmico (BLECHNER, 1993). Ao mesmo tempo, a redução na $\mathrm{pO}_{2}$ promove alterações nas atividades biofísicas fetais representadas pelo tônus fetal, movimentos somáticos, movimentos respiratórios e reatividade cardíaca. O comprometimento destas variáveis não é simultâneo, pois os centros que regulam estas atividades biofísicas têm sensibilidades diferentes a hipóxia e a acidemia (RURAK \& GRUBER, 1983). 
A presença de acidose fetal, aferida ao nascimento pelos valores de $\mathrm{pH}$ dos vasos umbilicais, é considerada como o melhor marcador para diagnóstico do sofrimento fetal (RURAK \& GRUBER, 1983; VINTZILEOS et al, 1991, MIYADAHIRA, 1997, ROLLAND DE SOUZA, 2008 ).

Valores de $\mathrm{pH}$ na artéria umbilical inferiores a 7,20 significam acidemia e sofrimento fetal e, portanto, falha no objetivo primordial da assistência obstétrica que é assegurar o bem estar do recém-nascido. É imprescindível antecipar possíveis situações de comprometimento do bem-estar fetal, lançando mão de outros recursos diagnósticos, visto que a avaliação do pH fetal é uma propedêutica invasiva, com riscos para o feto (ROLLAND DE SOUZA, 2008).

A evolução na propedêutica fetal, pelo desenvolvimento de métodos que proporcionaram o estudo não invasivo da anatomia e dos aspectos fisiológicos da saúde fetal, tem permitido avanço extraordinário no entendimento da fisiopatologia fetal (VINTZILEOS et al., 1986; WLADMIROFF et al.,1992). O Doppler das artérias uterinas é o melhor preditor de resultados perinatais em gestantes com pré-eclâmpsia (MELER et al, 2009).

\section{5- Labetalol e sua ação anti-hipertensiva}

A gestação é um estado fisiológico associado com alterações na disposição cinética e no metabolismo de fármacos. O clearance não renal e renal de medicamentos é aumentado na gestação, podendo traduzir-se em menores concentrações plasmáticas de medicamentos quando gestantes são tratadas com dosagens tradicionais (LOEBSTEIN et al., 1997). 
Os $\beta$-bloqueadores atenolol e labetalol, são efetivos agentes anti-hipertensivos, empregados em todos os tipos de hipertensão da gravidez (MAGGE et al., 2000; DAWES, 2001; MAGGE, 2001; ELATROUS et al, 2002).

O labetalol (5- [ 1 - hidroxi - 2- (1 - metil - 3 - fenilpropilamino) etil ] hidrocloreto salicilamida) é um dos anti-hipertensivos de primeira escolha na gravidez, uma vez que aumenta o fluxo útero placentário em decorrência da redução da resistência vascular uterina. No entanto, apresenta variabilidade inter paciente na dose requerida para controle da pressão sanguínea (ELATROUS et al., 2002).

Esse anti-hipertensivo age como bloqueador seletivo $\alpha_{1}$ adrenérgico e não seletivo $\beta$ adrenérgico, com proporção $\alpha / \beta$ de 1:7 após administração intravenosa e 1:3 após administração oral. Reduz a resistência vascular sistêmica mantendo os fluxos sanguíneos cerebral, renal e coronariano. Pode ser administrado em dose inicial de $20 \mathrm{mg}$, seguida de doses de manutenção de 20-80mg administradas a cada 10 minutos até o alcance da pressão sanguínea desejada (VARON \& MARIK, 2003).

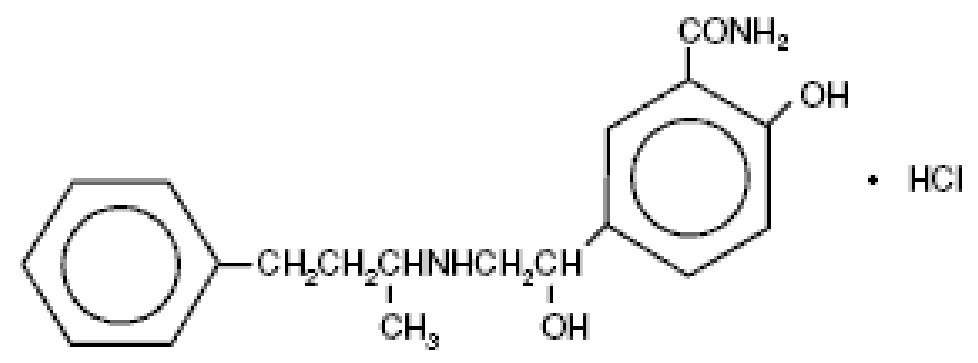

Figura 1: Estrutura química do labetalol. 
O labetalol é metabolizado principalmente no fígado, com menos de $5 \%$ da dose sendo excretada sob a forma inalterada na urina. Sofre acentuado metabolismo pré sistêmico, resultando em baixa biodisponibilidade oral, em torno de $30 \%$. No sangue do cordão umbilical, as concentrações plasmáticas do labetalol representam 50\% a $75 \%$ das concentrações plasmáticas observadas no sangue materno no momento do parto. O labetalol é excretado no leite materno com pico de concentração em 3 horas após a dose (GOEL et al., 2004).

\section{1-6 Hidralazina e sua ação anti-hipertensiva}

A hidralazina é uma substância ativa derivada quimicamente do 1-hidralazinoftalazina, que age diretamente na musculatura lisa, com predominância ativa sobre os vasos de alta resistência (pequenas artérias e arteríolas) e menor efeito nos vasos de capacitância (pequenas veias e vênulas). Resguarda a oxigenação de áreas vitais, como a esplâncnica, coronariana, cerebral e renal. Não apresenta ação cardíaca direta, sendo que a queda da pressão arterial estimula barorreceptores que reforçam a atividade simpática, resultando no aumento da freqüência cardíaca, do volume de ejeção sistólica, do débito cardíaco e maior oxigenação do miocárdio (SATO et al., 2003; ARCE et al., 2006).

A absorção dessa droga por via oral é rápida e eficaz (início de ação em 3 horas, pico em 6 horas e absorção de $65 \%$ a 90\%). A via endovenosa é a mais utilizada nas emergências hipertensivas da gestação, sendo o início e o pico de ação em 10 e 30 minutos respectivamente, persistindo por três a oito horas em pacientes com função renal normal (LUBBE, 1984). 
A hidralazina é rapidamente distribuída no organismo, sendo excretada através da urina. Após 24 horas da ingestão oral, aproximadamente $80 \%$ da mesma pode ser recuperada na urina. Atravessa a barreira placentária e é excretada através do leite materno (LOEBSTEIN, 1997; MONTAN, 2004).

O mecanismo de ação celular responsável pelo efeito de vasodilatação periférica da hidralazina não é totalmente conhecido, porém sabe-se que determina redução da pressão arterial (maior na diastólica do que na sistólica) e aumento da frequência cardíaca, do volume de ejeção e do débito cardíaco. A vasodilatação periférica é difusa mas não uniforme, aumentando o fluxo sanguíneo renal, cerebral, coronariano e esplâncnico, a não ser quando a queda da pressão arterial é muito acentuada. Uma vez que a hidralazina não apresenta propriedades cardiodepressoras ou simpatolíticas, os mecanismos regulatórios reflexos produzem aumento no volume de ejeção e da frequência cardíaca (GOODMAN AND GILMAN'S, 1996)

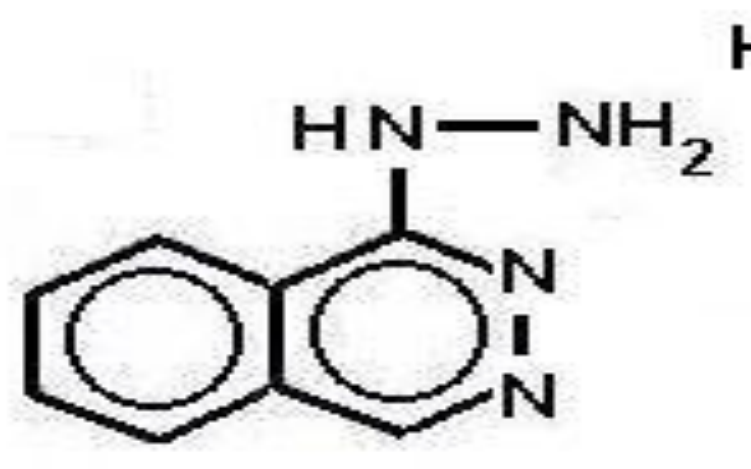

Figura 2: Estrutura química da hidralazina. 
Os efeitos colaterais mais comuns (taquicardia, hipotensão postural, cefaléia, vômitos e rubor facial) são secundários a vasodilatação causada pela medicação (CORDOVIL, 2003).

Outros efeitos colaterais são causados por reações imunológicas, sendo a reação lupuslike a mais comum. Existem evidências da relação do uso da hidralazina com alterações de metilação do ácido desoxirribonucléico (DNA), o que têm resultado em pesquisas na área de oncologia. O uso crônico da hidralazina pode produzir reação Coombs positiva, anemia hemolítica auto-imune, síndrome reumatóide-like e síndrome antifosfolípide (ARCE et al., 2006).

Em 1980, Pritchard consagrou o uso de hidralazina nas crises hipertensivas de gestantes, estabelecendo doses de 5mg a 10mg a cada 15-20 minutos até o controle dos níveis da pressão arterial. Geralmente, a administração entre 5 e 20mg é suficiente para atingir pressão arterial diastólica em torno de 90mmHg (GUTIERREZ et al., 2002; CORDOVIL, 2003; VASCONCELLOS, 2004).

Pritchard et al (1984), em 245 casos consecutivos de eclâmpsia, utilizando a hidrazalina como droga hipotensora associada ao sulfato de magnésio, obtiveram um dos melhores índices de sobrevida materna e fetal na história do tratamento de eclâmpsia.

\subsection{Justificativa}

A ultra-sonografia com Doppler é amplamente empregada na avaliação materno-fetal em gestações de alto risco, ressaltando-se aquelas complicadas com hipertensão, contribuindo com a redução da mortalidade perinatal (WESTERGAARD et al, 2001). 
Nas gestantes com Síndrome Hipertensiva a avaliação hemodinâmica materno fetal, através do estudo Doppler, representa a principal metodologia para avaliação da vitalidade fetal (VINTZILEOS, 1991; NOMURA, 2001).

No momento de crise hipertensiva ocorre vasoconstrição arteriolar generalizada com diminuição do fluxo útero placentário e consequentemente déficit do aporte de oxigênio nas áreas de troca materno fetal, submetendo o feto a regime de hipóxia transitória com alterações na avaliação dopplervelocimétrica.

A administração de anti-hipertensivo aumenta o fluxo útero placentário, em decorrência da redução da resistência vascular uterina, restaurando o fluxo útero placentário e determinando mudanças na avaliação da oxigenação fetal, através da metodologia Doppler (WILLIAMS \& WILSON, 1999). 


\section{Objetivos}

Avaliar as condições hemodinâmicas materno-fetais durante o período da crise hipertensiva e a resposta hemodinâmica materno-fetal após o tratamento com labetalol e/ou hidralazina. 


\section{3-PACIENTES E MÉTODOS}

\subsection{Pacientes}

\subsubsection{Aspectos éticos do projeto:}

O trabalho intitulado "ESTUDO HEMODINÂMICO MATERNO FETAL PRÉ E PÓS-TRATAMENTO DE CRISE HIPERTENSIVA" foi analisado pelo Comitê de Ética em Pesquisa do HC-FMRP, em sua $220^{\underline{a}}$ Reunião Ordinária realizada em 06 de março de 2006, sob o número 15416/2005 e enquadrado na categoria APROVADO, bem como o Termo de Consentimento Livre e Esclarecido - TCLE (Anexo A).

Todas as pacientes que participaram da pesquisa consentiram e assinaram o Termo de Consentimento Livre e Esclarecido (Anexo B).

Ressalta-se que a pesquisa não interferiu na conduta clínica a ser adotada no caso, sendo que a evolução obstétrica da paciente seguiu o protocolo do Departamento de Ginecologia e Obstetrícia da FMRP-USP. Todas as pacientes foram informadas, de forma clara e objetiva, sobre o protocolo de pesquisa a ser executado, com adesão espontânea ao projeto e foi obtido o consentimento livre e esclarecido de todas as pacientes que participaram do projeto. Ainda ficou estabelecido que caso ocorressem intercorrências, como aparecimento de efeitos colaterais inesperados ou indesejáveis, automaticamente a mesma seria afastada do protocolo de pesquisa e manteria seu tratamento garantido no HCFMRP-USP. 


\subsubsection{Seleção de pacientes}

Foram avaliadas gestantes hipertensas em crise hipertensiva, com idade inferior a 45 anos e idade gestacional entre 32 e 40 semanas, atendidas no Centro Obstétrico do Hospital das Clínicas da Faculdade de Medicina de Ribeirão Preto - USP.

\section{Critérios de Inclusão:}

- $\quad$ Ser portadora de hipertensão arterial em qualquer de suas formas e ter mais de 32 semanas de gestação comprovadas pela data da última menstruação e confirmadas pela ultrasonografia.

- Ter diagnóstico de crise hipertensiva definida por valor de pressão arterial maior ou igual a 160x110 mmHg, em duas medidas consecutivas, com intervalo de 30 minutos, aferida em posição sentada (Ministério da Saúde, 2000).

\section{Critérios de Exclusão:}

- Apresentar sintomas de iminência de eclâmpsia.

- Ser portadora de: diabete melito descontrolado (com hemoglobina glicosilada acima de $10 \mathrm{mg} \%$ ), obesidade mórbida (IMC $>40 \mathrm{Kg} / \mathrm{m}^{2}$ ) ou de insuficiência cardíaca congestiva

- Ter antecedente de infarto ou acidente vascular cerebral há menos de 06 meses; insuficiência renal crônica ou história de doença pulmonar obstrutiva crônica grave.

- Alteração de exames de vitalidade fetal (cardiotocografia, perfil biofísico fetal dooplervelocimetria fetal) 


\subsubsection{Tipo de estudo}

Foi realizado um estudo transversal tipo coorte, entre os anos de 2007 e 2008 em gestantes hipertensas com diagnóstico de crise hipertensiva, atendidas no Centro Obstétrico do Hospital das Clínicas da Faculdade de Medicina de Ribeirão Preto/USP.

\subsection{Métodos}

\subsubsection{Identificação das pacientes}

Todas as pacientes foram identificadas no momento do estudo, pela coleta de dados relativos à idade, paridade, idade gestacional, antecedentes pessoais patológicos, classificação da hipertensão e tratamento realizado.

\subsubsection{Avaliação perinatal}

Foram coletados dados relativos à via de parto, idade gestacional, sexo, peso, e Apgar dos recém nascidos.

\subsubsection{Classificaçao da forma de manifestação da hipertensão arterial}

Quanto à hipertensão, as pacientes foram classificadas de acordo com as diretrizes do National High Blood Pressure Education Program (2000) em: 
- Hipertensão arterial crônica (essencial ou secundária)

- Pré-eclâmpsia

- Pré- eclâmpsia sobreposta

- Hipertensão gestacional

\subsubsection{Avaliação hemodinâmica materna}

A pressão arterial materna foi aferida por meio de esfigmomanômetro digital Wrist Type Blood Pressure Monitor, modelo KP-6120 (Jump Health Co. Ltda, Taipei Hsien, Tawain) no pulso não dominante e expressa em mmHg e a frequência cardíaca expressa em batimentos por minuto.

Esses parâmetros foram avaliados antes, durante e após o tratamento da crise hipertensiva. As pacientes foram submetidas a exame de USG obstétrica com avaliação dopplervelocimétrica de artérias uterinas, cerebral média fetal e umbilical durante um episódio de crise hipertensiva e após o controle da pressão arterial.

A crise hipertensiva foi considerada controlada quando a paciente apresentou valores de pressão arterial menores que 150/100mmHg.

\subsubsection{Tratamento da crise hipertensiva}

Para tratamento da crise hipertensiva foram utilizados labetalol e/ou .hidralazina.

A dose inicial de labetalol foi de $20 \mathrm{mg}$, repetida a cada 10 minutos até controle da pressão arterial, com dose máxima utilizada de $80 \mathrm{mg}$. 
A hidralazina foi utilizada na dose inicial de $5 \mathrm{mg}$ repetida a cada 15 minutos até controle da pressão arterial ou dose máxima de $20 \mathrm{mg}$.

\subsubsection{Avaliação ultrassonográfica e dopplervelocimétrica}

Os exames de USG com avaliação dopplervelocimétrica materna e fetal foram realizados em uma sala no Laboratório de Ginecologia e Obstetrícia do HCFMRP-USP em ambiente tranqüilo, com temperatura refrigerada em torno de $23^{\circ} \mathrm{C}$ e iluminação suave. Antes da avaliação ultra-sonográfica as gestantes ficaram em repouso por um período de 10min, em posição de Fowler.

Os exames USG foram realizados com equipamento ATL, Philips, modelo HDI 3500 (Advanced Technologies Laboratories, USA), dotado de Doppler pulsátil, Doppler colorido e Doppler de amplitude (Power Doppler). Foi utilizado transdutor convexo, com freqüência de 2 a $5 \mathrm{MHz}$ e filtro de $50 \mathrm{~Hz}$, para avaliação das artérias uterinas, umbilical e cerebral média fetal. O limite de amostra foi de $3 \mathrm{~mm}$ para artérias uterinas e umbilical e de $1 \mathrm{~mm}$ para artéria cerebral média e o ângulo de insonação foi menor que $60^{\circ}$.

O exame foi conduzido por um investigador encarregado de realizar a avaliação ultrassonográfica com estudo Doppler materno e fetal após constatar pressão arterial maior ou igual a 160x110mmHg. Após a primeira avaliação ultrassonográfica a paciente foi medicada com labetalol e/ou hidralazina (endovenosos) até se obter níveis de pressão arterial abaixo de 150x1000mmHg, quando foi repetido o estudo Doppler materno e fetal. 
Todas as pacientes foram orientadas a respirar espontaneamente durante a avaliação ultrasonográfica e não realizarem manobras de Valsalva, para evitar respostas reflexas da frequiência cardíaca materna, devido ao aumento da pressão intratorácica induzida por esta manobra. (EKHOLM \& ERKKOLA, 1996).

A análise dopplervelocimétrica foi realizada em períodos de repouso fetal, diante de frequência cardíaca fetal normal e ausência de movimentos respiratórios, e os seguintes vasos foram avaliados: AUs direita e esquerda, AUm e ACM, seguindo essa seqüência no exame.

Para avaliação das AUs o transdutor foi colocado no quadrante inferior lateral direito do abdome e angulado medialmente. O Doppler colorido foi utilizado para identificar a artéria uterina homolateral ao transdutor, no ponto de cruzamento com a artéria ilíaca externa e a amostra de volume foi colocada a aproximadamente a $1 \mathrm{~cm}$ do ponto de cruzamento.

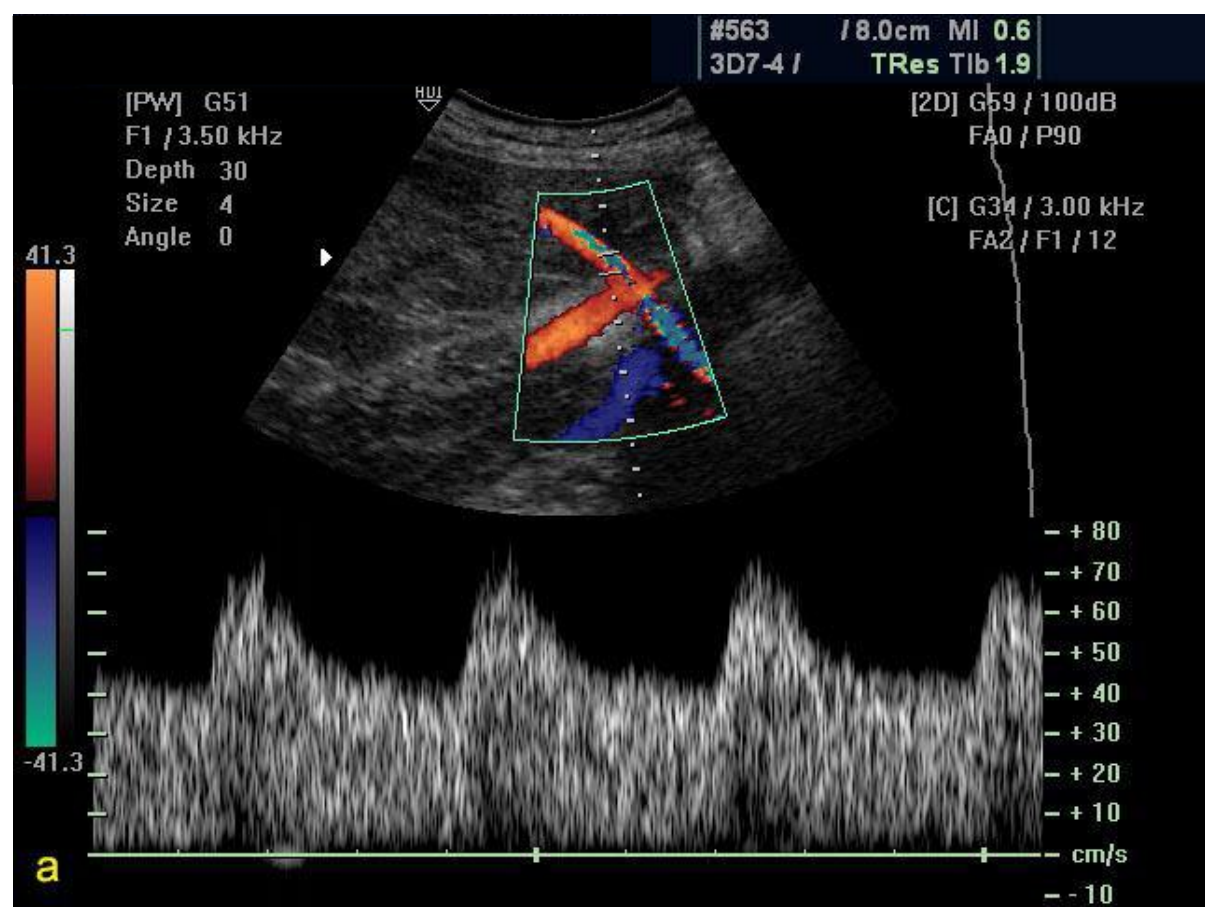

Figura 3: Dopplervelocimetria color e pulsada da artéria uterina. 
A artéria umbilical foi identificada com Doppler colorido e avaliada em alça livre de cordão.

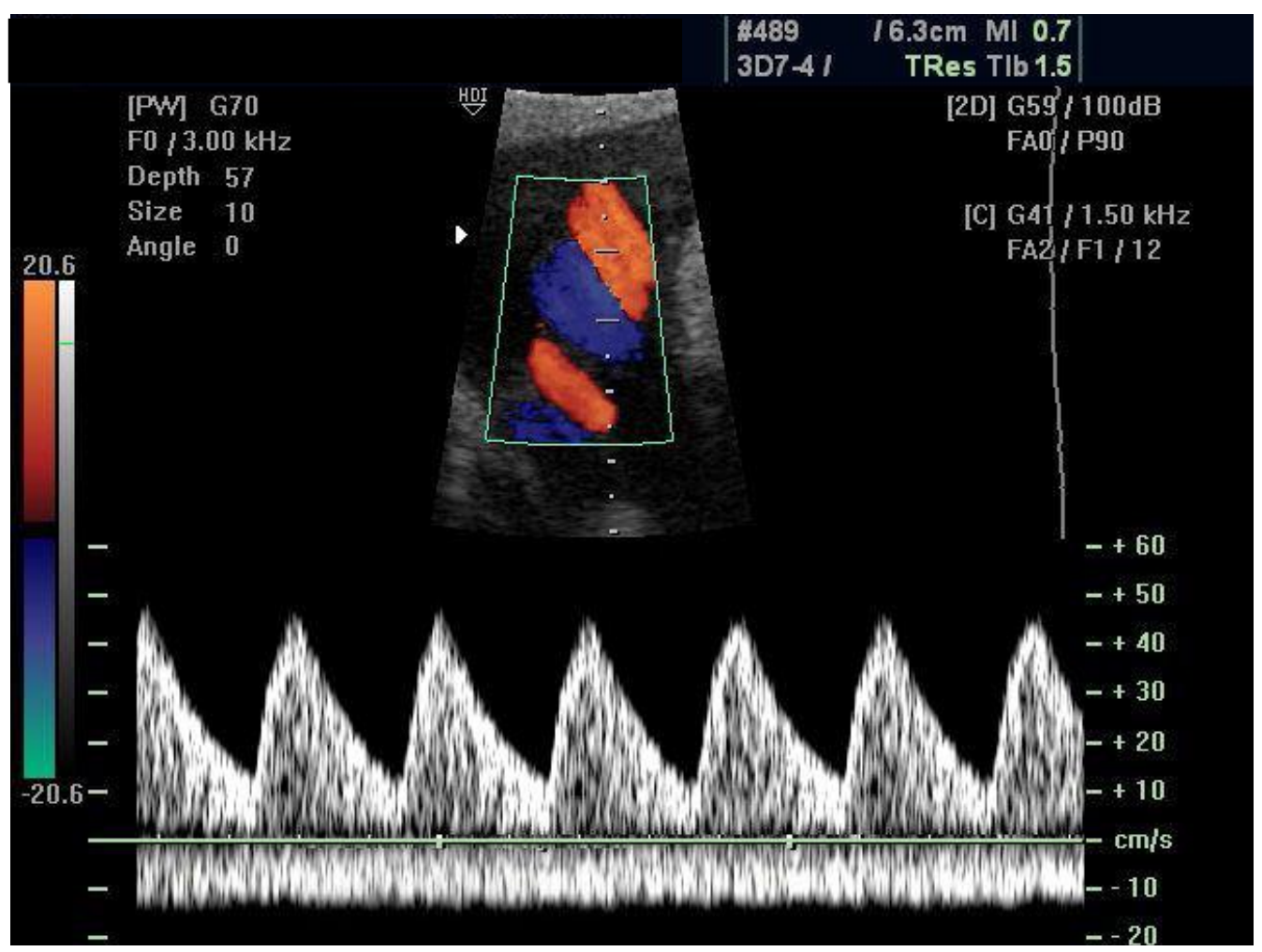

Figura 4: Dopplervelocimetria color e pulsada da artéria umbilical.

Para a aquisição do traçado Doppler da ACM foi feito, inicialmente, um corte axial da cabeça fetal no nível do tálamo e cavo do septo pelúcido. Em seguida moveu-se o transdutor até observar-se, por inclinação cranial, o polígono de Willis e a pulsação das duas artérias cerebrais médias. Com auxílio do Doppler de amplitude pode-se mapear todo o trajeto da artéria a ser estudada, acionando-se em seguida o Doppler espectral. O indicador da amostra foi calibrado para um volume de amostra de $1 \mathrm{~mm}$ e colocado na ACM, o mais próximo da calota craniana, antes de sua bifurcação, local onde provavelmente ocorrem as primeiras modificações resultantes das alterações hipóxicas útero placentárias (LUZI et al., 1996) 

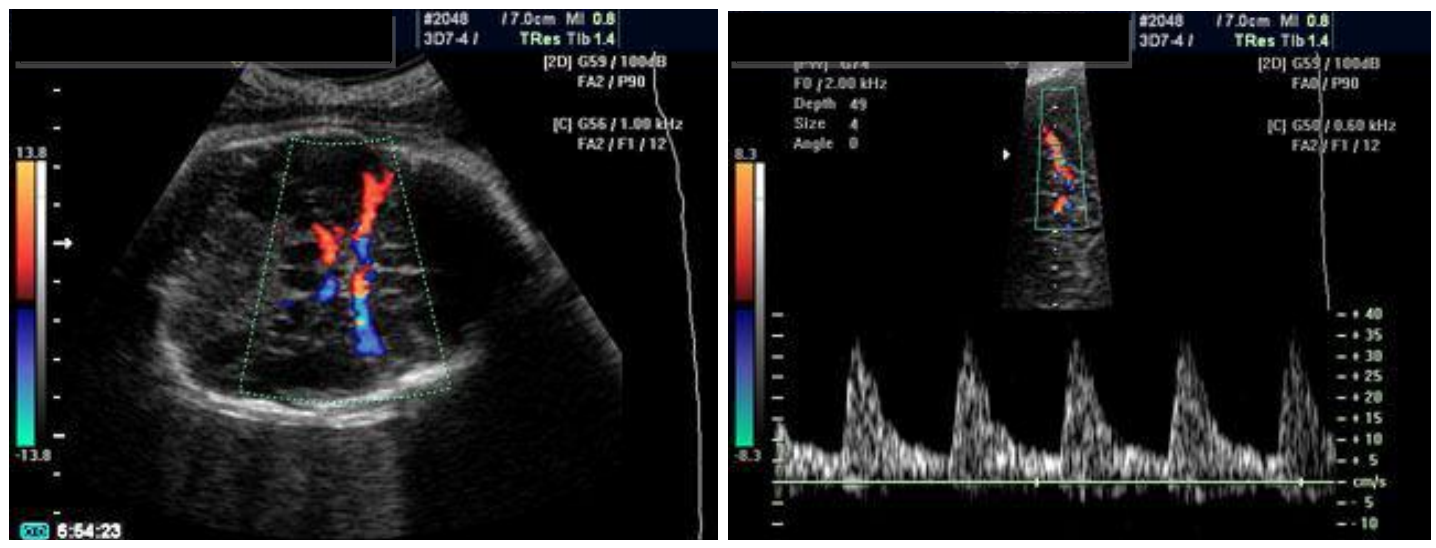

Figura 5: Dopplervelocimetria color e pulsada da artéria cerebral média.

As medidas Doppler dos vasos analisados foram realizadas após obtenção de, pelo menos, cinco ondas de velocidade de fluxo similares e de qualidade satisfatória.

Os seguintes parâmetros foram utilizados para análise do estudo Doppler:

- Índice de pulsatilidade (IP): (velocidade sistólica - velocidade diastólica)/velocidade média (GOSLING, 1975).

-Índice de resistência (IR): (velocidade sistólica - velocidade diastólica)/velocidade sistólica (POURCELOUT, 1974).

-Relação sístole/diastóle (S/D): velocidade sistólica/ velocidade diastólica (DEVOE, 1992).

Foram considerados valores normais para a artéria umbilical: relação S/D - até 2,99 e índices de resistência e de pulsatilidade com valores inferiores ao percentil 95, constantes nas tabelas utilizadas pelo Harris Birthright Research Center for Fetal Medicine e para a artéria cerebral média: índices de pulsatilidade menores que o limite inferior definido para normalidade, de acordo com os valores estipulados por Mari e Deter (1992). 
O IR, também chamado de índice de Pourcelout, atinge seu valor máximo, nos casos de fluxo diastólico ausente, representando resistência vascular muito alta. Ao contrário, quanto maior o fluxo diastólico, menor o índice e portanto maior a perfusão do órgão de estudo (BURNS, 1987).

\subsection{Análise estatística}

Para a análise estatística utilizou-se o software SPSS 16 for Windows (SPSS Inc., Chicago, Illinois, EUA).

A análise estatística foi feita por meio da Análise de Variância (ANOVA) para comparações entre as semanas por meio de contrastes e Análise de Regressão.

A Análise de Variância (ANOVA) foi feita com intuito de verificar se havia diferença nos valores dos índices estudados. Para quantificar essa relação foram realizadas comparações entre os dados pré e pós crise hipertensiva.

Por meio da Análise de Regressão foi aplicado aos dados obtidos, o ajuste quadrático e o linear, permitindo demonstrar de forma gráfica o comportamento dos parâmetros Doppler utilizados para artérias uterinas, cerebral média fetal e umbilical na crise hipertensiva e após o controle da pressão arterial.

Em todas as análises estatísticas foi utilizado como nível de significância p $<0,05$. 


\section{4-Resultados}

\section{1- Características maternas}

Foram avaliadas 18 gestantes durante e após controle de crise hipertensiva, atendidas no Centro Obstétrico do HCFMRP-USP, onde foram submetidas à análises dopplervelocimétricas, para avaliação de perfil hemodinâmico materno-fetal pré e pós tratamento da crise, nos anos de 2007 e 2008. A idade média das gestantes foi de 29,11 anos, com desvio padrão de 7,83, sendo os extremos etários de 17 e 44 anos. Os exames foram realizados em média com 242dias (34,5sem) de idade gestacional, com variações mínima e máxima, de 224 e 266 dias respectivamente.

Em relação a paridade, $28 \%$ das gestantes eram nulíparas e $72 \%$ multíparas. De acordo com a classificação proposta pelo NHBPEP (2000), 52,9\% das pacientes eram portadoras de hipertensão arterial crônica e 64,4\% portadoras de pré-eclâmpsia.

\subsection{Avaliação do quadro hipertensivo}

Em relação à classificação do quadro hipertensivo gestacional, as pacientes foram divididas em quatro grupos denominados: hipertensão arterial crônica, pré-eclâmpsia, préeclâmpsia sobreposta e hipertensão gestacional, cujos dados estão listados na tabela 1. 
Tabela 1: Classificação da hipertensão de acordo com a paridade (n:18).

\begin{tabular}{lcccc}
\hline Paridade/SHG & HAC & PE & HAC+PE & HG \\
\hline G1 & & 2 & 1 & 2 \\
G2/G3 & 3 & 3 & 3 & \\
$>$ G3 & $\mathbf{3}$ & $\mathbf{6}$ & 3 & $\mathbf{2}$ \\
Total & $\mathbf{1 7}$ & $\mathbf{3 3}$ & $\mathbf{3 9}$ & $\mathbf{1 1}$ \\
$\mathbf{\%}$ & & & & \\
\hline
\end{tabular}

Nota: G1- primigesta, G2/G3-secundigesta/tercigesta, >G3 multípara, \%-porcentagem

As crises hipertensivas foram tratadas com labetalol em 8 casos $(44,4 \%)$, hidralazina em 9 casos $(50 \%)$ e labetalol e hidralazina em 1 caso $(5,6 \%)$. A dose média utilizada de labetalol foi de 40mg e a dose média de hidralazina foi de $11,67 \mathrm{mg}$, com doses mínimas e máximas de 5 e 20mg respectivamente (Tabela 2).

Tabela 2: Tipo de hipertensão e tratamento da crise hipertensiva (n:18).

\begin{tabular}{lccc}
\hline & Labetalol(L) & Hidralazina(H) & L+H \\
\hline HAC & 2 & 1 & \\
PE & 3 & 3 & 1 \\
HG & 1 & & \\
HAC+PE & 2 & 5 & 1 \\
Total & 8 & 9 & \\
\hline
\end{tabular}

Notas :HAC-hipertensão arterial crônica, PE- pré-eclâmpsia, HG- hipertensão gestacional 
Os exames laboratoriais realizados nas avaliações maternas durante as crises hipertensivas estão apresentados na tabela 3

Tabela 3: Avaliação laboratorial de gestantes com crise hipertensiva (n:18).

\begin{tabular}{lcccc}
\hline \multicolumn{1}{c}{ Parâmetros } & Unidade & Media & Desvio padrão & Valores de \\
& & & & referência \\
\hline Uréia & $\mathrm{mg} / \mathrm{dL}$ & 20,20 & 9,10 & $10-50$ \\
Creatinina & $\mathrm{mg} / \mathrm{dL}$ & 0,72 & 0,16 & $0,5-1,2$ \\
Sódio & $\mathrm{mEq} / \mathrm{L}$ & 137,11 & 3,20 & $135,0-145,0$ \\
Potássio & $\mathrm{mEq} / \mathrm{L}$ & 4,13 & 0,40 & $3,5-5,0$ \\
TGO & $\mathrm{U} / \mathrm{L}$ & 20,78 & 11,40 & $7-39$ \\
Bilirrubina Total & $\mathrm{mg} / \mathrm{dL}$ & 0,41 & 0,34 & $<1,2$ \\
Bilirrubina Direta & $\mathrm{mg} / \mathrm{dL}$ & 0,11 & 0,10 & $<0,4$ \\
Hemoglobina & $\mathrm{g} / \mathrm{dL}$ & 11,83 & 1,30 & $12-18$ \\
Plaquetas & $10^{3} / \mathrm{mm}{ }^{3}$ & 219,66 & 76,00 & $150-450$ \\
Proteinuria 24 hs & $\mathrm{mg} \% / \mathrm{ml}$ & 1125,73 & 2465,20 & negativa \\
\hline
\end{tabular}

Nota: TGO-transaminase glutâmico oxalacética

A pressão arterial sistólica média na crise hipertensiva foi de $174,71 \mathrm{mmHg}$ e a pressão arterial diastólica média de 112,35mmHg. 


\subsection{Avaliação dopplervelocimétrica}

Os parâmetros Doppler avaliados na crise hipertensiva foram: IP da AUD 1,24 $\pm 0,42$, IP da AUE 1,29 \pm 0,40, IR da ACM de 0,78 \pm 0,06 e IR da AUm de 0,65 \pm 0,12. Após o tratamento da crise hipertensiva a pressão arterial sistólica média foi de $146,47 \mathrm{mmHg}$ e a pressão arterial diastólica média de 87,06 mmHg. Os parâmetros doppler avaliados após a crise hipertensiva foram: IP da AUD 1,26 \pm 0,37, IP da AUE 1,37 $\pm 0,36$, IR da ACM de 0,78 $\pm 0,09$ e IR da AUm de $0,67 \pm 0,09$

Tabela 4: Média e desvio padrão de parâmetros avaliados pelo Doppler das artérias uterinas direita e esquerda na população de gestantes estudada (n:18).

\begin{tabular}{|c|c|c|c|c|c|}
\hline & Direita & & Esquerda & & \\
\hline & Média & $\mathrm{DP}$ & Média & $\mathrm{DP}$ & $\mathrm{p}$ \\
\hline IP Inicial & 1,24 & 0,42 & 1,29 & 0,40 & 0,64 \\
\hline IP Final & 1,26 & 0,37 & 1,37 & 0,36 & 0,44 \\
\hline IR Inicial & 0,62 & 0,10 & 0,64 & 0,09 & 0,41 \\
\hline IR Final & 0,64 & 0,11 & 0,67 & 0,08 & 0,50 \\
\hline
\end{tabular}


Tabela 5: Média e desvio padrão da pressão arterial e dos parâmetros avaliados pelo Doppler materno/fetal na população de gestantes estudada $(\mathrm{n}=18)$.

\begin{tabular}{lccccc}
\hline & Inicial & \multicolumn{2}{c}{ Final } & & \\
& Média & DP & Média & DP & P \\
\hline Pressão arterial sistólica & 174,71 & 20,35 & 146,47 & 12,22 & $<0,01$ \\
Pressão arterial diastólica & 112,35 & 5,62 & 87,06 & 4,70 & $<0,01$ \\
IP da art. umbilical & 1,08 & 0,28 & 1,13 & 0,24 & 0,32 \\
IR da art. umbilical & 0,65 & 0,12 & 0,67 & 0,09 & 0,54 \\
IP da art. cerebral média & 1,53 & 0,24 & 1,65 & 0,43 & 0,25 \\
IR da art. cerebral média & 0,78 & 0,06 & 0,78 & 0,09 & 0,82 \\
IP médio das art. uterinas & 1,27 & 0,32 & 1,32 & 0,24 & 0,47 \\
IR médio das art. uterinas & 0,63 & 0,08 & 0,66 & 0,06 & 0,24 \\
\hline
\end{tabular}

Notas: IP = índice de pulsatilidade; IR = índice de resistência; DP = desvio padrão da média; valor de p obtido pelo teste t pareado. Para o valor de IR e IP das artérias uterinas foi considerado a média entre o valor obtido na artéria uterina direita e esquerda.

\subsection{Avaliação dos resultados perinatais}

Quanto à via de parto, 38,9\% evoluíram para parto vaginal e 61,1\% para cesareana. Em relação ao recém-nascido, o peso médio foi de $2262 \mathrm{~g}$ e os índices de Apgar de $1^{\circ}$ minuto menores que 7 em $17 \%$ dos casos e índices de Apgar de $5^{\circ}$ minuto menores que 7 em $0,55 \%$ dos casos. 
Tabela 6: Média e desvio padrão de parâmetros gestacionais e resultados perinatais avaliados na população de gestantes estudada $(n=18)$.

\begin{tabular}{|c|c|c|c|c|}
\hline Parâmetros & Média & $\mathbf{D P}$ & $\mathbf{N}$ & $\%$ \\
\hline Parto vaginal & & & 7 & 38,9 \\
\hline Parto cesárea & & & 11 & 61,1 \\
\hline Idade gestacional & 248 & 13,3 & & \\
\hline Peso & 2262,5 & 366,7 & & \\
\hline Apgar $1^{\circ} .<7$ & & & 3 & 17 \\
\hline Apgar $1^{\circ} .>7$ & & & 15 & 83 \\
\hline Apgar $5^{\circ} .<7$ & & & 1 & 0,55 \\
\hline Apgar 50. 77 & & & 17 & 9,45 \\
\hline
\end{tabular}




\section{Discussão}

Segundo dados do Ministério da Saúde, as desordens hipertensivas da gestação representam a primeira causa de morte materna no Brasil. Sabe-se ainda que, pré-eclâmpsia e eclâmpsia são responsáveis por mais de 50000 mortes maternas por ano no mundo, sendo a segunda causa de morte materna nos EUA (VIGIL DE GRACIA et al., 2006).

Sete a dez por cento de todas as gestações são afetadas pela hipertensão, incidência que pode variar de acordo com a população estudada e com os critérios utilizados para diagnóstico (PERAÇOLI \& RUDGE, 2000). Destas pacientes, 30\% são hipertensas crônicas e o restante desenvolve pré-eclâmpsia/eclâmpsia e hipertensão gestacional (AFIFI, 2003).

No presente estudo, $33 \%$ das pacientes apresentaram pré-eclâmpsia e $11 \%$ hipertensão gestacional, enquanto 56\% das pacientes eram hipertensas crônicas, sendo que destas 39\% desenvolveram pré-eclampsia associada. Considerando que, a pré-eclâmpsia, isolada ou superposta à hipertensão arterial crônica é quem determina o resultado materno e perinatal, no presente estudo a pré-eclâmpsia manifestou-se em 72,2\% dos casos. Destas, 33,3\% apresentaram pré-eclâmpsia isolada e 38,9\% pré-eclâmpsia superposta à hipertensão arterial crônica.

O estudo foi realizado em momentos de crise hipertensiva e após o tratamento da mesma. A crise hipertensiva pode manter-se como urgência, em que a ausência de comprometimento de órgãos-alvo permite o controle pressórico em até 24 horas ou iniciar-se e/ou evoluir para emergência, quando o controle pressórico deve ser rápido, em até 1 hora. 
O tratamento da hipertensão é indicado quando a pressão arterial apresenta-se maior ou igual a 160x110mmHg e tem como objetivo proteger contra lesão de órgãos alvo As drogas de ação imediata para controle da crise hipertensiva devem reduzir a pressão arterial diastólica em cerca de 20\%, mantendo desta forma níveis de pressão arterial diastólica que não comprometam o fluxo útero placentário (VIGIL-DE GRACIA et al., 2006).

A impossibilidade de previsão na evolução do quadro impõe como medida mínima a observação da gestante por 12 horas, em ambiente tranqüilo sob terapia anti-hipertensiva aguda e controle de dados vitais e vitalidade fetal. Idealmente preconiza-se a internação (Ministério da Saúde, 2002).

Diante da gravidade dos quadros hipertensivos na gestação, é imprescindível que vitalidade materna e fetal sejam adequadamente preservadas. Durante muito tempo, o óbito materno foi considerado um fato natural e inerente à condição feminina. Segundo dados do Ministério da Saúde (2002), 98\% desses óbitos seriam evitáveis caso fossem asseguradas condições dignas de vida e de saúde à população.

O controle adequado dos níveis pressóricos através do uso de medicações antihipertensivas é uma das principais preocupações diante de uma gestante com síndrome hipertensiva.

O tratamento farmacológico ideal deve objetivar reduzir a pressão arterial à níveis considerados seguros para a manutenção adequada da circulação feto placentária, com o mínimo possível de efeitos colaterais, utilizando drogas de baixo custo e pelo menor tempo possível (MAGEE, 2001)

Magee et al. (2003) realizaram revisão sistemática utilizando 21 ensaios clínicos randomizados, que empregaram a hidralazina no controle da crise hipertensiva da gestação. 
Esses estudos envolveram 893 gestantes, sendo que oito deles compararam essa droga com a nifedipina e cinco compararam com o labetalol. A hidralazina esteve mais associada a: hipotensão materna (risco relativo - $R R=3.29$ ), maior número de cesarianas $(R R=1.30)$, maior risco de descolamento prematuro de placenta $(R R=4.17)$, maior incidência de oligúria $(R R=4.00)$ e maior incidência de Apgar baixo $(R R=2.70)$. Os autores afirmam que não existem ainda evidências que indiquem a hidralazina como escolha de primeira linha para o controle da crise hipertensiva. Sugerem que estudos controlados mais profundos devam ser conduzidos comparando o fármaco com a nifedipina e o labetalol.

Estudo realizado por Montan (2004), afirma que a hipertensão grave deve ser tratada sem demora, reduzindo riscos maternos agudos. Neste trabalho, no entanto, o autor postula que as drogas não evitam a pré-eclâmpsia e a mortalidade perinatal, além de não inverter a curva de peso nos casos de crescimento intra-uterino restrito. Admite a hidralazina como droga de primeira escolha, porém considera que a nifedipina não deve ser descartada.

Drogas vasoativas como labetalol, hidralazina, pindolol, nifedipina ou anestésicos não interferem na circulação útero placentária e fetal e não trazem complicações clínicas. Algumas mudanças regulatórias ocorrem, no entanto, nas artérias fetais, cerebral e renal e na contratilidade miocárdica fetal. Isto significa que algumas drogas vasoativas podem comprometer a hemodinâmica fetal, especialmente em fetos com baixa reserva de $\mathrm{O}_{2}$ (JOUPPILLA, 1995).

Existe evidência que o labetalol (combinação de alfa e beta-bloqueador) é relativamente seguro e eficaz na gestação (SIBAI et al., 1987, 1990; BARRON et al., 1990).

Neste estudo utilizou-se hidralazina em $50 \%$ das pacientes, labetalol em $45 \%$ e labetalol e hidralazina em 5\%. Nenhuma pacientes apresentou alteração de vitalidade fetal ou instabilidade hemodinâmica materna secundária à medicação utilizada. 
As gestações de alto risco caracterizadas genericamente por um potencial comprometimento vascular, como acontece com as síndromes hipertensivas, podem evoluir com déficit de função placentária, às vezes de início precoce, necessitando de seguimento diferenciado no que se refere ao produto conceptual (ANDRADE, 2002).

No momento de crise hipertensiva ocorre vasoconstrição arteriolar generalizada com diminuição do fluxo útero placentário, e consequentemente alterações dopplervelocimétricas. O tratamento com anti-hipertensivo aumenta o fluxo útero placentário em decorrência da redução da resistência vascular uterina, restaurando o fluxo útero placentário (WILLIAMS \& WILSON, 1999).

A dopplervelocimetria em Obstetrícia é utilizada para o acompanhamento das gestações de alto risco, estudando a função placentária e a resposta fetal à hipoxemia. Hoje, tornou-se exame de rotina obrigatório, auxiliando na decisão de qual a melhor conduta obstétrica a ser adotada (CAMANO; SASS; MORON, 2006).

Alterações dos parâmetros Doppler permitem a detecção não invasiva e direta de insuficiência placentária e de centralização fetal, que ocorre como um mecanismo de adaptação à hipoxemia crônica. Essas alterações são de grande interesse na prática obstétrica, pois podem antecipar-se ao sofrimento fetal. Alterações significativas nos parâmetros Doppler sugerem que, mesmo após a redistribuição do sangue na centralização fetal, mantendo-se a hipoxêmia, ainda ocorrem alterações hemodinâmicas, como o aumento dos valores do índice de pulsatilidade da artéria cerebral média, decorrentes de diminuição da produção local de agentes. 
A avaliação da vitalidade fetal através do Doppler é fundamental nas pacientes com quadros hipertensivos (FERRAZZI et al., 1989)

O Doppler de artéria uterina é capaz de identificar, entre as gestantes portadoras de hipertensão arterial crônica, um subgrupo sujeito a elevadas taxas de pré-eclâmpsia (FRUSCA et al., 1998).

O índice de resistência da artéria umbilical, que reflete a resistência placentária, diminui gradualmente ao longo da gravidez. Entretanto essa diminuição é mais evidente após 32 semanas, quando a estrutura placentária esta completa (STUART, 1989).

Fong et al., em 1996, consideraram o IP menos suscetível a erro aleatório que o IR e presumivelmente mais sensível. Nos Estados Unidos, porém, a relação S/D tem sido o índice de Doppler mais utilizado e aceito para detectar alterações de vitalidade fetal.

Gojnic et al. (2004) aceitam que a dopplerfluxometria obstétrica seria o método ideal para avaliação do bem estar fetal, pois representa com fidedignidade a realidade da circulação uteroplacentária e fetal. Afirmam que o método é claro em diminuir os riscos perinatais, mas aumenta o índice de cesarianas.

Os resultados dopplervelocimétricos correlacionam-se com resultados perinatais adversos. Uma análise dos índices sístole/diástole das artérias uterinas e umbilical deve fazer parte da avaliação clínica de todas as mulheres grávidas com hipertensão. Isso resultará na elaboração de protocolos de tratamento mais eficientes e uma melhoria clínica significativa (DUCEY J. et al, 1987). 
A dopplervelocimetria tornou-se uma ferramenta importante na avaliação e condução de casos de gravidez de alto risco. As alterações dopplervelocométricas de artérias umbilicais e uterinas têm sido relacionadas à gestações complicadas pela hipertensão arterial e restrição do crescimento fetal (GUPTA, 2009).

No presente estudo as drogas utilizadas para crise hipertensiva foram o labetalol e a hidralazina, fármacos de ação rápida, que não causam quedas abruptas na pressão arterial diastólica (MAGEE et al., 2007). As pacientes apresentavam níveis de pressão arterial diastólica média de $110 \mathrm{mmHg}$ durante a crise hipertensiva e após tratamento os níveis médios apresentados foram de $87 \mathrm{mmHg}$, mostrando queda média de $22 \%$ nos valores da pressão arterial diastólica. Desta forma, a manutenção dos parâmetros dopplervelocimétricos após o tratamento da crise hipertensiva, sugere que o tratamento objetivando queda controlada da pressão arterial diastólica não afeta o fluxo útero placentário e portanto não modifica a hemodinâmica fetal.

O fluxo útero placentário pode ser monitorado pela análise dopplervelocimétrica das artérias uterinas. No presente estudo, observou-se que apesar da queda da pressão arterial sistólica e diastólica de $16 \%$ e $22 \%$ respectivamente, não houve comprometimento dos índices de pulsatilidade e resistência média das artérias uterinas.

A manutenção do fluxo útero placentário estável durante a crise hipertensiva, não alterou a hemodinâmica fetal, avaliada através da análise de fluxo da artéria umbilical e da artéria cerebral média, que apresentaram média dos parâmetros Doppler sem diferença estatística após o tratamento da crise hipertensiva. 
O diagnóstico de insuficiência placentária pela dopplervelocimetria das artérias umbilicais alterada, quando associado ao diagnóstico de centralização fetal, apresenta pior prognóstico perinatal. Nas fases iniciais do comprometimento da função placentária nem sempre o feto apresenta-se em situação de centralização da circulação. O diagnóstico de centralização realmente alerta quanto à possibilidade do comprometimento da oxigenação fetal, porém o intervalo até a ocorrência deste último fato é variável e deve sempre ser ponderado, principalmente nos casos de prematuridade extrema (NOMURA, 2001).

De acordo com o presente trabalho, não houve diferenças estatísticas significativas nos parâmetros avaliados pelo doppler quando comprados os valores na crise hipertensiva e após o controle da pressão arterial. Esses resultados são compatíveis com a literatura (PIRHONEN, 1991; OLIVEIRA, 2002).

Segundo o estudo de Pirhonen (1991) em gestantes normotensas, com 38 semanas de idade gestacional, que receberam labetalol endovenoso, não foram observadas alterações nos parâmetros dopplervelocimétricos fetais.

Estudo envolvendo 35 gestantes portadoras de hipertensão arterial crônica, que avaliou o índice de resistência da artéria umbilical, o índice de resistência da artéria cerebral média e frequiência cardíaca fetal, após tratamento de crise hipertensiva fetal com nifedipina sub lingual, não encontrou diferença significante entre os parâmetros dopplerfluxométricos realizados antes, 10 e 30 minutos após o tratamento (OLIVEIRA, 2002). 
As gestações de pacientes com síndromes hipertensivas são consideradas de alto risco e , portanto, devem ser alvo de observação rigorosa durante o pré-natal. A análise dopplervelocimétrica fetal (artérias cerebral média e umbilical) e materna (artérias uterinas) deve ser realizada para avaliação da vitalidade fetal nessas pacientes, inclusive durante crise hipertensiva. 


\section{Conclusões}

Este estudo demonstrou não haver diferença na avaliação dos parâmetros dopplervelocimétricos materno e fetal, durante e após crise hipertensiva tratada com labetalol e/ou hidralazina. 


\section{Referências Biblográficas}

AALI, B. S.; NEJAD, S. S. Nifedipine or hydralazine as a first-line agent to control hypertension in severe preeclampsia. Acta Obstet Gynecol Scand 2002; 81(1): 25-30.

American College of Obstetrics and Gynecologists. Hypertension in pregnancy. ACOG Tech

Bull 1996; 219: 1-8.

ACOG. Diagnosis and management of preeclampsia and eclampsia. ACOG Practice Bulletin No.

33. Obstet Gynecol 2002; 99: 159-67.

AFIFI, Y.; CHURCHILL, D. Pharmacological treatment of hypertension in pregnancy. Curr Pharm Des.2003; 9(21): 1745-1753.

ALTON, K. B.; CHAN, T.; PRAMANIK, B. N. Urinary metabolites. Diabetes Care 2003; 26(1): S33-S50.

ANDRADE, J. Q.; MIYADAHIRA, S.; NOMURA, R. M. Y.; FRANCISCO, R. P. V.; ZUGAIB, M. Dopplervelocimetry of the Arterial and Venous Compartments of the Fetal and Umbilical Circulation in High-Risk Pregnancy - Perinatal Results. RBGO 2002; 24(3): 153-160. 
ARCE, C.; SEGURA-PACHECO, B.; PEREZ-CARDENAS, E.; TAJA-CHAYEB, L.; CANDELARIA, M.; DUENNAS-GONZALEZ, A. Hydralazine target: From blood vessels to the epigenome, Journal of Translational Medicine 2006; 4:10

BASCHAT, A. A.; GEMBRUCH, U.; REISS, I.; GORTNER, L.; WEINER, C. P.; HARMAN, C. R. Relationship between arterial and venous Doppler and perintal outcome in fetal growth restriction. Ultrasound Obstet Gynecol 2000; 16: 407-413.

BASCHAT, A. A.; GENBRUCH, U.; WEINER, C. P.; HARMAN, C. R. Qualitative venous Doppler waveforms analysis improve prediction of critical perinatal outcomes in premature growth restricted fetuses. Ultrasound Obstet Gynecol 2003; 22: 240-245.

BERNAREGGI, A. Farmacocinetica di principi attivi chirali. Boll. Chim. Farm.1995;.134: 638.

BLECHNER, J. N. Maternal-fetal acid-base physiology. Clin Obstet Gynecol 1993; 36:3-12.

BURNS, P. N. The physical principles of Doppler and spectral analysis. J. Clin. Ultrasound 1987; 15(9): 567-90.

CAETANO, M. et al. A survey of Canadian practitioners regarding the management of the hypertensive disorders of pregnancy. Hypertension in Pregnancy 2004;:23(1):.61-74. 
CAMANO, L.; SASS, N.; MORON, A .F. Hipertensão arterial na gestante. SBM 2006; 1(1): 3540.

CERRI, G .G.; MÓLNAR, L .J.; VEZOZZO, D. C. P. Doppler., Sarvier, 1996; 1-30.

CORDOVIL, I. Hipertensão na gravidez, Editorial Revista Hospital de Laranjeiras 2003; 1:.2.

DAWES, M. Pharmacokinetics in pregnancy, Best Practice \& Research Obstetrics and Gynaecology 2001; 15(6): 819-826.

DELMIS, J. Hypertension in pregnancy., Lijec Vjesn. 2006 Nov-Dec;128(11-12):357-68.

DEVOE, L. D.; GARDNER, P.; DEAR, C.; FAIRCLOTH, D. The significance of increasing umbilical artery systolicdiastolic ratios in third-trimester pregnancy. Obstet Gynecol 1992; 80: 684-7.

DUCEY, J.; SCHULMAN, H.; FARMAKIDES, G.; ROCHELSON, B.; WINTER, D.; PENNYB BRACERO, A.; FLEISHER, A.; GUZMAN, E. A classification of hypertension in pregnancy based on Doppler velocimetry. Am J Obstet Gynecol. 1987 Sep;157(3):680-5.

EKHOLM, E. M.; ERKKOLA, R. U. Autonomic cardiovascular control in pregnancy. European Journal of Obstetrics, Gynecology, and Reproductive Biology 1996;64(1): 29-36. 
ELATROUS, S.; NOUIRA, S.; BESBES, O.; MARGHLI， S.; BOUSSARSSAR, M.; SAKKOUHI, M.; ABROUG, F. Short-term treatment of severe hypertension of pregnancy: prospective comparison of nicardipine and labetalol. Intensive Care Medicine.2002; 28(9): $1281-1286$.

FERRAZZI, E.; BELlOTTI, M.; VEGNI, C.; BARBERA, A.; PERUTA, S. D.; FERRO, B.; AGOSTONI, G.; PARDI, G.; Umbilical flow waveforms versus fetal biophysical profile in hypertensive pregnancies. European Journal of Obstetrics, Gynecology, and Reproductive Biology 1989; 33(3): 199-208.

FITZGERALD, D. E.; DRUMM, J. E. Non invasive measurement of human fetal circulation using ultrasound: a new method. Br. Med. J.1977; 2(6100): 1450-1.

FRUSCA, T.; SOREGAROLI, M.;ZANELLI, $\quad$ S.;DANTI， L.;GUANDALINI， F.; VALCAMONICO, A. Role of uterine artery Doppler investigation in pregnant women with chronic hypertension, European Journal of Obstetrics \& Gynecology and Reproductive Biology 1998; 79(1): 47-50.

GOJNIC, M. et al. The importance of Doppler ultrasound in delivery planning. Clin Exp Obstet Gynecol, 2004; 31(4): 282-4. 
GOLDABER, K. G.; GILSTRAP, L. C.; LEVENO, K. J.; DAX, J. S.; MCINTIRE, D. D. Pathologic fetal acidemia. Obstet Gynecol 1991; 78:1103-1106.

GOLDBERG, B. B. Obstetric US imaging: the past 40 years. Radiology 2000; 215(3):622-29.

GOODMAN AND GILMAN'S, The Pharmacological Basis of Therapeutics 1996, 9th. ed., The Mc-Graw-Hill.

GOSLING, R. G.; KING, D. H. Ultrasound angiology. In: Marcus AW \& Adamson J, Editors. Arteries and Veins 1975; Edinburgh: Churchill-Livingstone: 61-71.

GUPTA, U.; QURESHI, A.; SAMAL, S. Doppler Velocimetry In Normal And Hypertensive Pregnancy. The Internet Journal of Gynecology and Obstetrics 2009; 11: 2.

GUTIÉRREZ, A. et al. Protocolo de actuación ante la preeclampsia. Nuestra experiência. Acta Obst Ginec 2002; 19: 1.

HOFMAN, C.; GALAN, H. L.; Assessing the 'at-risk' fetus: Doppler ultrasound. Curr Opin Obstet Gynecol. 2009; 21(2): 161-6.

JOUPPILA, P.; RASANEN, J.; ALAHUNTA, S.; JOUPPILA, R. Vasoactive Drugs in Obstetrics: A Review of Data Obtained by Doppler and Color Doppler Methods: Invited Reviews. Hypertension in Pregnancy 1995; 14(3): 261-275. 
KANEKO, Z. First steps in the development of the Doppler flowmeter. Ultrasound Med. Biol 1986; 12(3): 187-95.

LOEBSTEIN, R.; LALKIN, A.; KOREN, G. Pharmacokinetic changes during pregnancy and their clinical relevance. Clin Pharmacokinet 1997; 33(5): 328-343.

LUBBE, W. F. Hypertension in pregnancy-pathophysiology and management. Drugs 1984; 28: $170-188$.

LUZI, G.; COATA, G.; CASERTA, G.; RENZO, G. Doppler velocimetry of different sections of the fetal middle cerebral artery in relation to perinatal outcome. Journal of Perinatal Medicine 1996; 24(4): 327-334

McCALLUM, W. D. Qualitative estimation of blood velocity changes in human umbilical arteries after delivery. Early Hum Dev 1977; 1(1): 99-106.

MAGGE, L. A.; ELRAN, E.; BULL, S. B.; LOGAN, A.; KOREN, G.; Risks and benefits of $\beta$ recptor blckets for pregnancy hypertension: overview of the randomized trials. European Journal of Obstetrics, Gynecology and Reproductive Biology, 2000; 88: 15-26.

MAGEE, L. A. Antihypertensives, Best Practice \& Research. Obstetrics and Gynaecology $2001 ; 15(6): 827-845$. 
MAGEE, L. A. et al. Hydralazine for treatment of severe hypertension in pregnancy: metaanalysis. Br Med J 2003; 327(7421): p.955-60.

MARI, G.; DETER, R. L. Middle cerebral artery flow velocity waveforms in normal and smallfor-gestational age fetuses. Am J Obstet Gynecol 1992; 166:1262-70.

MELER, E.; FIGUERAS, F.; MULA, R.; CRISPI, F.; BENASSAR, M.; GÓMEZ, O.; GRATACÓS, E. Prognostic Role of Uterine Artery Doppler in Patients with Preeclampsia. Fetal Diagn Ther 2009 (DOI: 10.1159/000258048).

MIKOVIC, Z.; MANDIC, V.; DJUKIC, M.; EGIC, A.; FILIMONOVIC, D.; CEROVIC, N.; POPOVAC, M. Longitudinal analysis of arterial Doppler parameters in growth retarded fetuses. Srp Arh Celok Lek 2003 ;131(1-2): 21-5.

Ministério da Saúde: Secretaria de Políticas de Saúde. Área Técnica da Saúde da Mulher. Manual dos Comitês de Mortalidade Materna. $2^{\mathrm{a}}$ ed. Brasília; 2002.

Ministério da Saúde: Secretaria de Políticas de Saúde. Área Técnica da Saúde da Mulher. Urgências e Emergências Maternas: guia para diagnóstico e conduta em situações de risco de morte materna $2^{\mathrm{a}}$ ed. Brasília; 2002. 
MIYADAHIRA, S. Avaliação da vitalidade fetal. In: Medicina Fetal 1997. Editores: Zugaib, M; Pedreira, DAL; Brizot, ML; Bunduki, V. $2^{\mathrm{a}}$ edição. Editora Atheneu, 479-485, São Paulo.

MONTAN, S. Drugs used in hypertensive diseases in pregnancy. Curr Opin Obstet Gynecol 2004; 16(2): 111-5.

NHBPEP-National High Blood Pressure Education Program Working Group on High Blood Pressure in Pregnancy. Am J Obstet Gynecol. 2000; 183(1): S1-S22.

NOMURA, R. M. Y.; FRANCISCO, R. P V.; MIYADAHIRA, K. S. S.; ZUGAIB, M. Redistribution of Fetal Circulation: Assessment of Fetal Well-Being and Perinatal Outcome in High Risk Pregnancies. Rev Bras Ginecol Obstet 2001; 23: 3.

ONWUDIWE, N.; YU, C. K.; POON, L. C.; SPILIOPOUlOS, I.; NICOLAIDES, K. H. Prediction of pre-eclampsia by a combination of maternal history, uterine artery Doppler and mean arterial pressure. Ultrasound Obstet Gynecol 2008; 32(7): 877-83.

OLIVEIRA, S. F. Estudo da Hemodinâmica Fetal por meio da Dopplervelocimetria nas Artérias Umbilical e Cerebral Média Durante a Crise Hipertensiva Materna antes e após o Tratamento com Nifedipina. Rev. Bras. Ginecol. Obstet 2002; 24: 9. 
PERAÇOLI, J. C.; RUDGE, M. V. C. Hipertensão arterial na gravidez. Rev Bras Med 2000; 57: $6-14$.

PIRHONEN, J. P.; ERKKOLA, R. U.;MAKINEN, J. I.; EKBLAD, U. U. Single Dose of Labetalol in Normotensive Pregnancy: Effects on Maternal Hemodynamics and Uterine and Fetal Flow Velocity Waveforms Biol Neonate 1991;59:204-208.

POURCELOUT, L. Applications cliniques de l'examen Doppler trascutané. In: Peronneau P, .Vélocimetrie ultrasonore Doppler 1974: 213-40.

PRITCHARD, J. A. The Parkland Memorial Hospital protocol for treatment of eclampsia. Am J Obstet Gynecol 1984; 147:.951-963.

RATH, W.; FISCHER, T. The diagnosis and treatment of hypertensive disorders of pregnancy: new findings for antenatal and inpatient care. Dtsch Arztebl Int. 2009; 106(45): 733-8.

ROLLAND DE SOUZA, A.; RAMOS AMORIM, M. Avaliação da vitalidade fetal intra-parto, Acta Med Port 2008; 21: 229-240.

ROLLAND DE SOUZA ET al, Efeito do sulfato de magnésio sobre o índice de pulsatilidade das artérias uterinas, umbilical e cerebral média fetal de acordo com a persistência da incisura protodiastólica da artéria uterina na pré-eclâmpsia grave, Rev Bras Ginecol Obstet. 2009; 31(2): $82-8$ 
RURAK, D.W.; GRUBER, N. C. The effect of neuromuscular blockade on oxigen consumption and blood gases in fetal lamb. Am J Obstet Gynecol 1983; 145: 258-262.

RYCHIK, J. Fetal cardiovascular physiology. Pediatr. Cardiol 2004; 25(3): 201-9.

SATOMURA, S. A study on examining the heart with ultrasonics. I. principles; II. Instrument. Jpn Circ J 1956; 20:227.

SATO, N.; TANAKA, K. A.; SZLAM, F.; TSUDA, A.; ARIAS, M. E.; LEVY, J. H. The Vasodilatory Effects of Hydralazine, Nicardipine, Nitroglycerin, and Fenoldopam in the Human Umbilical Artery, Anesth Analg 2003; 96:539-544.

SIBAI, B. M.; MABIE, W. C.; SHAMSA, F.; VILlAR, M. A.; ANDERSON, G. D.. A comparison of no versus methyldopa or labetalol in chronic hypertension during pregnancy. Am J Obstet Gynecol 1990; 162: 960-7.

SMITH, R. A.; BAKER, P. N. Risk factors, prevention and treatment of hypertension in pregnancy. Minerva Ginecol. 2005; 57(4): 379-88.

STUART, B.; DRUMM, J.; FITZGERALD, D.E.; DUIGNAN, N.M. Fetal blood velocity waveforms in normal pregnancy. Br J Obstet Gynecol 1980; 87: 780-5. 
TEKAY, A.; CAMPBELL, S. Ultra-sonografia com Doppler em Obstetrícia. In: CALLEN, P.W. Ultra-sonografia em Ginecologia e Obstetrícia, 2002. $4^{\text {a }}$ ed. Rio de Janeiro: Guanabara Koogan, 24, 637-81.

VARON, J.; MARIK, P. Clinical review: the management of hypertensive crises. Critical Care 2003; 7(5): 374-382.

VASCONCELlos, M. J. A. Pré-eclampsia. In: CHAVES NETTO, H. Obstetrícia Básica, 2004. Editora Atheneu, Rio de Janeiro, 1ed., 247.

VIGIL-DE GRACIA, P.; LASSO, M.; RUIZ, E. Severe hypertension in pregnancy: Hidralazine or labetalol. A randomizade clinical trial. European Journal Obstetrics, Ginecology and Reproductive Biology 2006; 128: 157-162.

VINTZILEOS, A. M.; CAMPBELL, W. A.; RODIS, J. F. Avaliação pré-natal por ultrasonografia: o perfil biofísico fetal. IN: CALLEN, PW Ultra-songrafia em Ginecologia e Obstetrícia, 1986. $3^{\text {a }}$ edição, Editora Guanabara Koogan, Rio de Janeiro, 473-487. 
VINTZILEOS, A. M.; FLEMING, A. D.; SCORZA, W. E. Relationship between fetal biophysical activities and umbilical cord blood gas values. Am J Obstet Gynecol 1991; 165: 707-713.

YOSHIMURA, S.; MASUZAKI, H.; MIURA, K.; GOTOH, H.; ISHIMARU, T. Fetal blood flow redistribution in term intrauterine growth retardation (IUGR) and post natal growth. Int. J. Gynaecol. Obstet 1998; 60(1): 3-8.

WESTGAARD, H. B.; LANGHOFF-ROOS, J.; LINGMAN, G.; MARSAL, K.; KREINER, S. A critical appraisal of the use of umbilical artery Doppler ultrasound in high-risk pregnancies: use of meta-analyses in evidence-based obstetrics. Ultrasound Obstet Gynecol 2001; 17(6): 464-5.

WILLIANS, K. P.; WILSON, S. Antepartum middle mean cerebral blood flow velocity correlation with maternal hemodynamics. Hypertension in Pregnancy 1999; 18(3): 273-8.

WITLIN, A. G.; SIBAI, B. M. Hypertension. Clin Obstet Gynecol 1998; 41: 533-44.

WLADIMIROFF, J. W.; WIJNGAARD, J. A.; DEGANI, S.; NOORDAM, M. J.; EYCK, J.; TONGE, H. M. Cerebral and umbilical arterial blood flow velocity waveforms in normal and growth-retarded pregnancies. Obstet Gynecol 1987; 69(5): 705-9. 
WLADMIROFF, J.W.; HUISMAN, T.W.A.; STEWART, P.A. Intracerebral, aortic and umbilical artery flow velocity wave forms in the late first trimestrer fetus. Am J Obstet Gynecol $1992 ; 166: 46-49$. 
Anexo A- Aprovação do Comitê de Ética em Pesquisa

HOSPITAL DAS CLINICAS DA FACULDADE DE MEDICINA
DE RIBEIRÃO PRETO DA UNIVERSIDADE DE SÃO PAULO
Comitê de Ética em Pesquisa

Ribeirão Preto, 09 de março de 2006

Ofício $\mathrm{n}^{\circ} 559 / 2006$

CEP/SPC

Senhor Professor:

O trabalho intitulado "ESTUDO HEMODINÂMICO MATERNO FETAL NA CRISE HIPERTENSIVA PRÉ E PÓs-TRATAMEnTo COM LABETALOL", foi analisado pelo Comitê de Ética em Pesquisa, em sua $220^{a}$ Reunião Ordinária realizada em 06/03/2006, e enquadrado na categoria: APROVADO, bem como o Termo de Consentimento Livre e Esclarecido, de acordo com o Processo HCRP no 15416/2005.

Aproveito a oportunidade para apresentar a Vossa Senhoria protestos de estima e consideração.

PROF. DR. SÉRGIO PEREIRA DA CUNHA

Coordenador do Comitê de Ética em Pesquisa do HCFMRP-USP C da FMRP-USP

Ilustríssimo Senhor

PROF. DR. RICARDO DE CARVALHO CAVALLI

Depto. de Ginecologia e Obstetrícia

Em mãos 
Anexo B

Termo de Consentimento Livre e Esclarecido

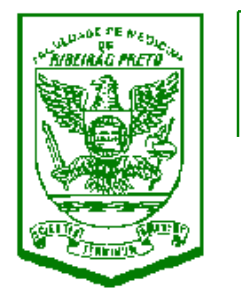

FACULDADE DE MEDICINA DE RIBEIRÃO PRETO-USP DEPARTAMENTO DE GINECOLOGIA E OBSTETRÍCIA

Av. Bandeirantes, 3900 - $8^{\circ}$ andar - Ribeirão Preto-SP - CEP 14049- 900

Fone (016) 633-0216/633-1028 - Fax (016) 633-0946

PESQUISA CIENTÍFICA

TERMO DE CONSENTIMENTO PÓS-INFORMAÇÃO

NOME DO PACIENTE / IDADE:

REGISTRO E/OU DOCUMENTO DE IDENTIFICAÇÃO:

NOME DA PESQUISA: Estudo hemodinâmico materno fetal na crise hipertensiva pré e pós-tratamento com anti-hipertensivo - labetalol

RESPONSÁVEIS CLÍNICOS: Prof. Dr Ricardo de Carvalho Cavalli 


\section{CONSENTIMENTO PÓS-INFORMAÇÃO}

Declaro que em .............................. concordei voluntariamente em participar, como paciente do projeto de pesquisa acima referido. Fui devidamente informado em detalhes pelo médico responsável que:

1- O estudo implica em que eu me submeta a procedimentos, exames complementares e tratamentos devidamente planejados, conforme texto a seguir:

"O nosso estudo visa entender como se comporta o fluxo de sangue na mãe e bebê durante a pressão alta e após o tratamento com medicação e avaliar os efeitos dos medicamentos utilizados para abaixar pressão arterial nas gestantes. Você receberá um comprimido de $200 \mathrm{mg}$ ou injeção de $40 \mathrm{mg}$ de um remédio usado para abaixar a pressão chamado de labetalol. Nós iremos neste mesmo dia realizar exame ultra-sonográfico da gestante e do bebê no momento da pressão arterial alta e após a melhora da pressão com uso de medicamento e paciente ficará internada em observação no Hospital das Clínicas.

A sua colaboração será importante para entendermos como ajustar a dose desse medicamento para controle da pressão arterial em outras gestantes hipertensas e avaliar como se comporta o bebê e as gestantes com a pressão alta.

2- Você não é obrigado a continuar participando do projeto e pode, a qualquer momento sair do mesmo, sem que isso impeço que você deixe de ser tratado como os demais pacientes do Hospital das Clínicas.

3- A pesquisa não será feita se houver, relacionados à mesma, grandes riscos para você. Da mesma forma, caso algum risco proibitivo venha a se revelar no decurso do estudo, o 
pesquisador se compromete a alertá-la sobre o fato e a suspender, de imediato, a sua participação como paciente.

4- Os pesquisadores garantem que responderão a qualquer pergunta ou farão esclarecimento a qualquer dúvida acerca dos procedimentos, riscos, benefícios e outros assuntos relacionados com a pesquisa e com o tratamento a que você será submetida.

5- Os pesquisadores se comprometem a proporcionar a você informações atualizadas durante o estudo, ainda que estas possam afetar a sua vontade de continuar participando da pesquisa.

6- Os pesquisadores se comprometem a manter o sigilo da sua participação e de publicar os resultados da pesquisa para a comunidade médica e científica pertinente.

6- Você terá direito a ressarcimento financeiro caso haja gastos gerados exclusivamente pela sua participação como voluntário desta pesquisa.

7- Caso haja dano comprovadamente decorrente da pesquisa você terá direito a indenização.

OBSERVAÇÕES COMPLEMENTARES:.

Ribeirão Preto, de de

Assinatura do paciente

Prof. Dr.

Ou de seu responsável

CRM........

\section{TESTEMUNHAS NÃO LIGADAS À PESQUISA}

$1-$

Assinatura

Identificação

$2-$

Assinatura

Identificação 